\title{
Qualidade do sono dos estudantes de medicina de uma faculdade do sul de Minas Gerais
}

\section{Quality of sleep among students at a medical school in Minas Gerais}

\author{
Glenia Junqueira Machado Medeiros' (D) gleniajmmedeiros@gmail.com \\ Pedro Fernandes Roma ${ }^{1}$ (D) pe_frm@hotmail.com

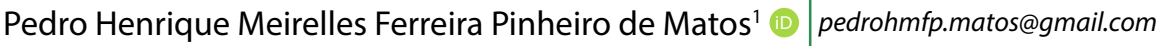

\section{RESUMO}

Introdução: O sono é um estado fisiológico que se dá de forma cíclica em grande quantidade de seres vivos do reino animal, tendo sido analisados comportamentos de repouso e atividade, que compõem um ciclo vigília-sono. Na universidade, os acadêmicos de Medicina se deparam com falta de tempo e com exaustão, visto que o curso possui carga horária integral, fato que faz com que os graduandos deixem as atividades básicas cada vez mais para o final do dia, o que os leva a desenvolver distúrbios do sono.

Objetivo: Este estudo teve como objetivo analisar a qualidade de sono e a incidência de distúrbio de sono em estudantes de Medicina.

Método: A pesquisa foi realizada individualmente com os estudantes de Medicina de uma faculdade do sul de Minas Gerais, via plataforma Google Forms, em que eles responderam a dois questionários autoaplicáveis. O primeiro englobava perguntas sobre gênero e ano de graduação, e o segundo, denominado Índice de Qualidade do Sono de Pittsburgh, avaliou a qualidade de sono. Foram utilizados os programas Bioestat 5 e Excel 365 para a análise estatística.

Resultado: A análise do estudo demonstrou distúrbio de sono em $20,5 \%$ dos estudantes e qualidade subjetiva de sono ruim ou muito ruim em $40,2 \%$ dos discentes.

Conclusão: Por meio da presente pesquisa, observou-se que a qualidade de sono dos estudantes de Medicina é inferior à da população geral, estando diretamente relacionada à progressão do curso. Foi concluído que os estudantes deste estudo têm, além disso, em média, menos horas de sono que o restante dos brasileiros.

Palavras-chave: Estudantes de Medicina; Privação do Sono; Transtornos do Sono-Vigília; Distúrbios do Início e da Manutenção do Sono.

\section{ABSTRACT}

Introduction: Sleep is a physiological state that occurs cyclically in many living beings of the animal kingdom, and the behaviours of rest and activity, that form the sleep-wake cycle, have been the subject of studies. Undergraduate medical students are faced with a lack of time and exhaustion, since the course entails full time study, which fact means that the students put off basic activities more and more until the end of the day, and thus develop sleeping disorders.

Objective: To analyze the quality of sleep and the incidence of sleeping disorders among medical students.

Method: The research was conducted individually with medical students from a college in the south of Minas Gerais, via the Google Forms platform, whereby the student answered two self-administered questionnaires. The first encompassed questions about gender and year of undergraduate study and the second assessed quality of sleep, referring to the Pittsburgh Sleep Quality Index. Bioestat 5 and Excel 365 programs were used for statistical analysis.

Result: Analysis of the study found sleep disturbance among 20.5\% of the students and poor or very poor quality of sleep among $40.2 \%$ of the students.

Conclusion: The research showed that the quality of sleep among medical students is lower than that of the general population, and is directly related to the progression of the course. It was concluded that the students in this study have, on average, fewer hours of sleep than the rest of Brazilians.

Keywords: Medical Students; Sleep Deprivation; Sleep-Wake Disorders; Sleep Onset and Maintenance Disorders.

${ }^{1}$ Faculdade de Medicina de Itajubá, Itajubá, Minas Gerais, Brasil.

Editora-chefe: Rosiane Viana Zuza Diniz.

Editor associado: Gustavo Antonio Raimondi.

Recebido em 10/05/21; Aceito em 30/09/21.

Avaliado pelo processo de double blind review. 


\section{INTRODUÇÃO}

O sono é um estado fisiológico que se dá de forma cíclica em grande quantidade de seres vivos do reino animal, tendo sido analisadoscomportamentosderepousoeatividade,quecompõem um ciclo vigília-sono. $O$ sono tem natureza não homogênea, visto que algumas fases dele apresentam características semelhantes às da vigília quando analisadas no eletroencefalograma (EEG), à medida que outras etapas demonstram ondas lentificadas no exame ${ }^{1}$. Desse modo, encontra-se dificuldade em definir de forma simplista o estado de sono.

A regulação do ciclo vigília-sono é feita nos núcleos supraquiasmáticos do hipotálamo, que controlam o ritmo circadiano no corpo ${ }^{2}$. Os núcleos recebem informação da quantidade de luz do ambiente. Essa informação permite sincronização do organismo com as 24 horas do dia geográfico 3 .

Geralmente o sono apresenta cinco fases: estágios I, II, III, IV (sono não-REM) e sono REM (rapid eye movement) ${ }^{4}$. No sono NREM, o indivíduo apresenta redução progressiva de movimentos corporais, aumento de ondas lentas no EEG e ausência de movimentos oculares rápidos. No sono REM, o indivíduo apresenta hipotonia ou atonia muscular, sonhos, movimentos oculares rápidos e movimentos físicos ${ }^{5}$.

Estudos realizados com seres humanos com privação de sono ou com distúrbios do sono demonstram comprometimento da atenção e de outras atividades cognitivas ${ }^{6}$. Longos períodos de privação do sono podem levar a delírios, desorganização do ego e alucinações ${ }^{7}$.

No Brasil, estima-se que de dez a 20 milhões de pessoas possuam distúrbios do sono ${ }^{8}$. Os distúrbios de maior prevalência na população geral são a insônia e a síndrome da apneia obstrutiva do sono?.

Os acadêmicos de Medicina, mesmo antes de adentrarem a universidade, enfrentam grande concorrência nos vestibulares e sofrem com alterações em atividades básicas como sono e alimentação ${ }^{10}$. Na universidade, os alunos de Medicina se deparam com falta de tempo e com exaustão nos primeiros períodos de estudo, visto que o curso possui carga horária integral.

Os discentes, ao buscarem uma boa qualificação profissional, complementam suas formações médicas com cursos extracurriculares deixando atividades básicas cada vez mais para o final do dia, o que os leva a desenvolver distúrbios do sono ${ }^{11}$.

Após a etapa inicial do curso, o aluno adentra a fase profissionalizante, na qual se depara com o paciente, a doença e a morte, seguida do ciclo de internato, período em que o estudante passa pela transição entre a vida acadêmica e de profissional médico, o que exige dedicação integral e dificuldade de controle do tempo ${ }^{12}$. Diversas publicações sobre privação de sono e consequente queda no rendimento profissional têm sido descritas na literatura ${ }^{13,14}$.

Além disso, no início do ano de 2020, os estudantes de Medicina foram expostos a um surto de doença causada pelo coronavírus da síndrome respiratória aguda severa 2 , a coronavirus disease 2019 (Covid-19). Rapidamente, o vírus se espalhou pelo mundo todo, tornando-se a primeira pandemia por coronavírus ${ }^{15}$.

A pandemia de Covid-19 provocou inúmeras mudanças na dinâmica de interação social, como isolamento social, receio de transmissão do vírus e adoção de novos métodos de ensino (alterações das atividades acadêmicas presenciais para remotas) ${ }^{16}$. Estudos indicam que tais mudanças geram consequências psicológicas, como maior índice de transtorno de ansiedade generalizada, transtorno de humor depressivo, baixa qualidade de sono, uso nocivo de drogas e menor bemestar mental, sobretudo quando se considera a população que não foi exposta à pandemia ${ }^{15,17}$.

O abuso do uso de medicamentos psicotrópicos sofre uma crescente no Brasil, sendo encontrado também entre os estudantes universitários ${ }^{18}$. Antidepressivos e ansiolíticos estão presentes de forma frequente no cotidiano dos acadêmicos que buscam induzir e manter o sono para aumentar o rendimento em atividades cotidianas e nos estudos. Portanto, torna-se necessário investigar como esses alunos estão dormindo, já que eles serão os futuros profissionais da saúde ${ }^{19}$ e o sono possui grande influência nas funções psíquicas do ser humano.

Diante do apresentado, os objetivos desta pesquisa incluem avaliar a qualidade do sono em alunos de Medicina de uma Faculdade de Medicina do sul de Minas Gerais, do primeiro ao sexto ano, e relacionar tal qualidade com alguns fatores possivelmente condicionantes (gênero e ano cursado).

\section{MATERIAIS E MÉTODOS}

Trata-se de estudo transversal descritivo realizado no período de janeiro a fevereiro de 2021. A população-alvo envolveu estudantes de graduação do curso de Medicina, do primeiro ao sexto ano de graduação, de ambos os sexos, devidamente matriculados no curso de Medicina de uma Faculdade de Medicina do sul de Minas Gerais. Foram critérios de exclusão para participação da pesquisa: estudantes do primeiro ao sexto ano da faculdade com menos de 18 anos, aqueles que estavam com a matrícula do curso trancada e discentes que não concordaram em participar do estudo.

Este estudo transversal envolve apenas os dados sobre qualidade do sono dos estudantes no início do semestre letivo. $\mathrm{O}$ estudo foi aprovado pelo Comitê de Ética em Pesquisa em Seres Humanos da instituição, sob o protocolo número 4.464.464. Todos os participantes assinaram o Termo de Consentimento 
Livre e Esclarecido (TCLE). Foi feito o cálculo amostral baseado em uma fórmula estatística em que o erro amostral é de $5 \%$ e o nível de confiança de $95 \%$, chegando assim a um valor amostral mínimo de 169 formulários a serem aplicados.

A pesquisa foi realizada individualmente com os estudantes de Medicina de uma Faculdade de Medicina do sul de Minas Gerais, via plataforma Google Forms, respeitando as necessidades de distanciamento social impostas pela pandemia da Covid-19. Na plataforma, os estudantes responderam a dois questionários autoaplicáveis. $\mathrm{O}$ primeiro englobava perguntas sobre gênero e ano de graduação, e o segundo, denominado Índice de Qualidade do Sono de Pittsburgh (Pittsburgh Sleep Quality Index - PSQI), avaliou a qualidade de sono. O link para preenchimento dos formulários foi enviado por meio de plataforma digital para os estudantes. Após o esclarecimento da pesquisa e obtenção do TCLE, os discentes responderam aos dois questionários.

OPSQI apresenta especificidade de $86,5 \%$ e sensibilidade de $89,6 \%$. Quando validado e traduzido para língua portuguesa, o PSQI apresentou uma especificidade discretamente menor, de $68,8 \%$, no entanto mantém uma significativa sensibilidade $(80 \%)^{20}$. O questionário é formado por dez questões, das quais duas são objetivas (5 e 10), e quatro (1, 2, 3 e 4), abertas.

O entrevistado tem a opção de escrever comentários no final das questões 5, 9 e 10, caso ele julgue pertinente. A interpretação do PSQI é feita a partir de um escore que apresenta sete componentes, e cada um recebe uma pontuação de zero a 3 . A pontuação máxima desse escore é de 21 pontos, na qual os escores superiores a 10 pontos são indicativos de distúrbio do sono; escores com resultados de 5 a 10 pontos, indicativos de qualidade ruim no padrão de sono ${ }^{21}$; e escores com resultados de zero a 4 pontos, indicativos de boa qualidade no padrão de sono.

Os componentes do PSQI são mensurados da seguinte maneira: o primeiro item compete à percepção individual a respeito da qualidade do sono, ou seja, refere-se à qualidade subjetiva do sono; o segundo trata da latência do sono, concernente à mensuração do tempo do momento que o estudante se deita no leito até iniciar o sono. $O$ terceiro corresponde à duração do sono (tempo que o entrevistado permanece dormindo); o quarto avalia a eficiência habitual do sono, verificado pela relação entre o número de horas em permanência na cama e o número de horas dormidas.

$O$ quinto item do PSQI verifica os distúrbios do sono. Nesse momento, analisam-se a frequência com que o estudante apresentou problemas de sono porque despertou no meio da noite ou de madrugada e a frequência com que teve problemas no sono por uma causa não descrita no questionário. $\mathrm{O}$ sexto item analisa o uso de medicação para dormir; o sétimo é referente aos distúrbios durante o dia e à sonolência diurna. No sétimo item, analisam-se a frequência com que os entrevistados apresentaram dificuldades em permanecer acordados enquanto dirigiam, faziam as refeições ou estavam envolvidos em atividades, e o grau de entusiasmo no último mês ao realizarem as atividades cotidianas no último mês.

Os dados recolhidos foram processados no banco de dados Excel 365, no qual foi realizada dupla digitação com o objetivo de corrigir os erros de transcrição, quando necessário. Além disso, realizou-se a confecção de gráficos e tabelas a partir desse programa. A análise estatística foi obtida com auxílio do programa Bioestat 5. Na análise dos dados quantitativos, utilizaram-se medidas do qui-quadrado e o teste de resíduo. Os valores foram considerados estatisticamente significantes quando $p=0,05$ /intervalo de confiança de $95 \%$.

\section{RESULTADOS}

Dos 269 estudantes que participaram deste estudo, 58 eram integrantes do primeiro ano de Medicina (21,60\%), 37 eram do segundo ano (13,8\%), 42 integravam o terceiro ano $(15,6 \%), 40$ eram do quarto ano $(14,9 \%), 48$ eram do quinto ano $(17,8 \%)$ e 44 estavam no sexto ano (16,3\%). Além disso, 103 estudantes que participaram do estudo se autodeclararam do sexo masculino $(38,29 \%)$ e 166 do sexo feminino $(61,71 \%)$.

Por meio do questionário PSQI, analisou-se que os estudantes demoravam, em média, 34,24 minutos para pegar no sono, com progressão do tempo com a crescente dos anos da graduação. A duração média do sono na amostra foi de 6,85 horas. Observou-se que $44,2 \%(n=119)$ dos estudantes classificaram a qualidade do seu sono no período do último mês como boa, $29,0 \%(n=78)$ a classificaram como ruim, $15,6 \%$ $(n=42)$ a classificaram como muito boa e $11,2 \%(n=30)$ a classificaram como muito ruim (Tabela 1).

A análise dos componentes do PSQI apontou que 47,2\% ( $n=127)$ dos discentes da amostra apresentavam qualidade ruim no padrão de sono, com diferentes prevalências entre os anos de formação médica do estudante, enquanto $32,3 \%$ ( $n=$ 87) demonstravam qualidade boa no padrão de sono e $20,5 \%$ $(\mathrm{n}=55)$ tinham distúrbio de sono. Não foram observadas

Tabela 1. Qualidade subjetiva do sono.

\begin{tabular}{ccc}
\hline Qualidade do sono & N & $\%$ \\
\hline Muito boa & 42 & 15,6 \\
Boa & 119 & 44,2 \\
Ruim & 78 & 29 \\
Muito ruim & 30 & 11,2 \\
\hline
\end{tabular}

Fonte: Elaborada pelos autores. 
diferenças estatisticamente significantes na qualidade do sono entre os sexos ( $p=0,5079)$, e, portanto, segundo o teste qui-quadrado de partição, tem-se que a qualidade de sono não está associada ao sexo (Tabela 2). Analisou-se o prérequisito e verificou-se que todos os valores esperados são maiores que 5, o que demonstrou ser possível utilizar o teste qui-quadrado.

No entanto, foram observadas diferenças estatisticamente significantes na qualidade do sono entre os estudantes de diferentes anos da graduação ( $p=0,0038)$, e, portanto, segundo o teste qui-quadrado de partição, tem-se que a qualidade de sono está associada ao ano de graduação, o que demonstrou ser possível utilizar o teste qui-quadrado (Tabela 3). Analisou-se o pré-requisito e verificou-se que todos os valores esperados são maiores que 5 , o que demonstrou ser possível utilizar o teste qui-quadrado.

Segundo o teste de resíduo, o terceiro ano apresentou uma boa qualidade de sono quando comparado aos outros anos com 95\% de confiabilidade. Já o quarto e quinto anos apresentaram mais distúrbio de sono quando comparados aos

Tabela 2. Qualidade do sono estratificada por sexo segundo resultados do PSQI.

\begin{tabular}{cccc}
\hline \multirow{2}{*}{ Qualidade do sono } & Feminino & Masculino & Total geral \\
\cline { 2 - 4 } & Freq. (\%) & Freq. $(\%)$ & Freq. $(\%)$ \\
\hline Boa & $51(30,7)$ & $36(35,0)$ & $87(32,3)$ \\
Ruim & $83(50,0)$ & $44(42,7)$ & $127(47,2)$ \\
$\begin{array}{c}\text { Presença de distúrbio } \\
\text { do sono }\end{array}$ & $32(19,3)$ & $23(22,3)$ & $55(20,5)$ \\
\hline
\end{tabular}

Fonte: Elaborada pelos autores. outros anos com 95\% de confiabilidade. Em relação ao sono ruim, não foi observada diferença significativa em relação aos anos (Tabela 4).

Entre os distúrbios do sono identificados na amostra pelo questionário PSQI inerentes ao mês anterior à entrevista, destaca-se o seguinte: $30,9 \%(n=83)$ dos estudantes tiveram problemas no sono porque despertaram no meio da noite ou de madrugada uma ou duas vezes por semana; $18,2 \%(n=49)$ precisaram levantar à noite para ir ao banheiro uma ou duas vezes por semana; $20,10 \%(n=54)$ relataram sonhos ruins três ou mais vezes por semana; e $39,7 \%(n=107)$ mencionaram que sentiram muito calor três ou mais vezes por semana.

Além disso, 13,7\% ( $n=37)$ dos participantes da amostra revelaram fazer uso de medicação para dormir. Dos entrevistados que fizeram comentários na questão 5 , $19,7 \%(n=53)$ apresentaram outras razões que ocasionavam problemas para dormir além daquelas propostas pelo questionário PSQI. Eis os principais resultados: 30,59\% ( $n=26)$ tiveram crises de ansiedade; $21,17 \%(n=18)$, dismenorreia; e 7,06\% ( $n=6)$, cefaleia no último mês.

$\mathrm{Na}$ questão 9, foi mensurada a sonolência diurna do entrevistado; e $27,9 \%(n=75)$ relataram moderada dificuldade de manter-se suficientemente entusiasmados a realizar as atividades diárias e $22,6 \%(n=61)$ mencionaram muita dificuldade de manter-se suficientemente entusiasmados a realizar as atividades diárias. Na questão 10, 16,3\% $(n=44)$ dos entrevistados relataram outras inquietações que apresentam durante o sono. Eis os principais resultados: $31,81 \%(n=14)$ apresentaram bruxismo; e 11,36\% ( $n=5)$, paralisia do sono no último mês (Tabela 5).

Tabela 3. Qualidade do sono estratificada por ano de graduação segundo resultados do PSQI.

\begin{tabular}{cccccccc}
\hline \multirow{2}{*}{ Qualidade do sono } & $\mathbf{1}^{\circ}$ ano & $\mathbf{2}^{\circ}$ ano & $\mathbf{3}^{\circ}$ ano & $\mathbf{4}^{\circ}$ ano & $\mathbf{5}^{\circ}$ ano & $\mathbf{6}^{\circ}$ ano & Total geral \\
\cline { 2 - 7 } & $\operatorname{Fr}(\%)$ & $\operatorname{Fr}(\%)$ & $\operatorname{Fr}(\%)$ & $\operatorname{Fr}(\%)$ & $\operatorname{Fr}(\%)$ & $\operatorname{Fr}(\%)$ & $\operatorname{Fr}(\%)$ \\
\hline Boa & $17(29,3)$ & $15(40,5)$ & $21(50,0)$ & $8(20,0)$ & $15(32,3)$ & $11(25,0)$ & $87(32,3)$ \\
Ruim & $33(56,9)$ & $18(48,7)$ & $19(45,2)$ & $19(47,5)$ & $17(35,4)$ & $21(47,7)$ & $127(47,2)$ \\
Distúrbio de sono & $8(13,8)$ & $4(10,8)$ & $2(4,8)$ & $13(32,5)$ & $16(33,3)$ & $12(27,3)$ & $55(20,5)$ \\
\hline
\end{tabular}

Fonte: Elaborada pelos autores.

Tabela 4. Resultado do teste de resíduo estratificado por ano de graduação segundo resultados do PSQI.

\begin{tabular}{|c|c|c|c|c|c|c|}
\hline & $1^{\circ}$ ano & $2^{\circ}$ ano & $3^{\circ}$ ano & $4^{\circ}$ ano & $5^{\circ}$ ano & $6^{\circ}$ ano \\
\hline Boa qualidade de sono & -0.5573 & 1.1480 & 2.6631 & -1.8086 & -0.1784 & -1.1384 \\
\hline Presença de distúrbio do sono & -1.4185 & -1.5648 & -2.7436 & 2.0487 & 2.4424 & 1.2277 \\
\hline Qualidade de sono Ruim & 1.6682 & 0.1885 & -0.2789 & 0.0396 & -1.8060 & 0.0749 \\
\hline Nível alfa 0.05 & 1.96 & & & & & \\
\hline Nível alfa 0.01 & 2.576 & & & & & \\
\hline
\end{tabular}

Fonte: Elaborada pelos autores. 
Tabela 5. Outras inquietações durante o sono no último mês segundo resultados do PSQI.

\begin{tabular}{ccc}
\hline Inquietações durante o sono & N & $\%$ \\
\hline Bruxismo & 14 & 31,81 \\
Paralisia do sono & 5 & 11,36 \\
Ansiedade & 3 & 6,87 \\
Sonambulismo & 2 & 4,54 \\
Espasmos & 2 & 4,54 \\
Pesadelos & 2 & 4,54 \\
Falar sozinho & 2 & 4,54 \\
Insônia & 2 & 4,54 \\
Cefaleia & 1 & 2,27 \\
Palpitações & 1 & 2,27 \\
Noctúria & 1 & 2,27 \\
Não especificada & 9 & 20,45 \\
\hline Total & 44 & 100 \\
\hline
\end{tabular}

Fonte: Elaborada pelos autores.

\section{DISCUSSÃO}

Diversos estudos têm ponderado a mensuração da qualidade de sono nos estudantes de Medicina. De acordo com esses estudos, os discentes de escolas médicas apresentam pobre qualidade de sono (PSQI > 5), num índice que varia de $44,59 \%$ a $87,1 \%{ }^{12,22-24}$, fato que corrobora o resultado da amostra, no qual $67,7 \%$ demonstraram pobre qualidade de sono.

A média geral de horas de sono dos estudantes da amostra foi de 6,85 horas diárias. Esse valor é inferior à média geral da população adulta brasileira (de sete a nove horas diárias) ${ }^{25}$, mas foi similar aos resultados encontrados em outros estudos também desenvolvidos com universitários brasileiros, europeus, estadunidenses e do Oriente Médio ${ }^{19,26}$. A quantidade média de horas dormidas por noite variou de 6,46 horas em estudantes do sexto ano a 6,97 horas em discentes do segundo ano.

Referente à latência do sono, os estudantes levaram em média 34,24 minutos para adormecer, valor superior a outros estudos em escolas médicas brasileiras ${ }^{20,27}$. 0 tempo médio para adormecer variou de 37,75 minutos em estudantes do quarto ano a 30,00 minutos em discentes do segundo ano.

Estudos apontam que a privação do sono pode acarretar uma redução da capacidade de se concentrar e aprender. Além disso, pessoas que dormem por menos horas podem ter impactos negativos em atividades sociais ${ }^{28}$, o que corrobora os resultados deste estudo, visto que $27,9 \%$ ( $n=75$ ) relataram moderada dificuldade de manter-se suficientemente entusiasmados a realizar as atividades diárias e $22,6 \%(n=61)$ mencionaram muita dificuldade de manter-se suficientemente entusiasmados a realizar as atividades diárias. Como consequência, outras funções cognitivas também serão prejudicadas, como a memória e a capacidade de tomar decisões ${ }^{29}$. O prejuízo das funções cognitivas é justificado pela redução do metabolismo no tálamo e córtex pré-frontal e parietal, e pela diminuição do fluxo de sangue cerebral ${ }^{30}$.

O teste de resíduo demonstrou que o terceiro ano apresentou uma boa qualidade de sono quando comparado aos outros anos. Esse fato se deve à adaptação dos estudantes desse período à carga horária extensa e à rotina de estudos exaustiva. Além disso, como esses discentes ainda não estão inseridos na prática clínica (geralmente exaustiva) e na rotina de internato, eles têm melhor qualidade do sono. Já o quarto e quinto anos apresentaram mais distúrbio de sono quando comparados aos outros anos. Esses dados são explicados pela maior carga horária destinada ao processo de formação médica e às atividades de prática clínica que já estão presentes nesses períodos.

Na amostra verificada neste estudo, não houve diferenças estatisticamente significativas entre os sexos ( $p=0,5079$ ). Essa constatação pode ser atribuída ao fato de que os indivíduos da amostra estudada estarem submetidos ao mesmo ambiente e às mesmas condições de exigência. No entanto, houve diferença estatística significante quando se relacionaram período do curso de medicina e qualidade do sono $(p=0,0038)$. Tal fato pode ser explicado pelas diferenças de horário de prática e teoria no decorrer do curso, e pelo ingresso dos estudantes no regime de internato, o qual insere o discentes na realidade da prática médica.

Vale lembrar que esses estudantes foram submetidos ao questionário no contexto da pandemia de Covid-19. Segundo um estudo chinês ${ }^{31}$, um terço dos estudantes de Medicina apresentou transtornos de ansiedade e baixa qualidade do sono durante a pandemia de Covid-19, não havendo diferença 
estatística de prevalência entre os sexos, o que corrobora o presente estudo.

Além disso, em um estudo italiano em que foi aplicado o PSQI, os participantes com baixa qualidade de sono eram 40,5\% antes da pandemia da Covid-19 e 52,4\% após a pandemia, o que correlaciona a baixa qualidade do sono à pandemia de Covid-1932.

Em outros estudos, foi relatado que adultos jovens têm maior probabilidade de apresentar depressão $0^{33,34}$, ansiedade ${ }^{33}$ e redução da qualidade do sono ${ }^{35}$ durante a pandemia de Covid-19. O presente estudo tem como amostra adultos jovens universitários, o que poderia justificar os altos índices de baixa qualidade de sono e distúrbios de sono. Uma possível explicação para esses altos índices se deve ao fato da necessidade desses estudantes de adaptação aos novos métodos de ensino na universidade ${ }^{36,37}$. Além disso, os atrasos nas atividades acadêmicas causados pela Covid-19 foram correlacionados com o aparecimento de transtorno de ansiedade generalizada ${ }^{38}$ e consequentemente distúrbios do sono.

Dos estudantes avaliados na amostra, 13,7\% ( $\mathrm{n}=$ 37) afirmaram tomar medicamentos para dormir. Essa taxa está dentro dos valores encontrados na literatura (8,7\%$18,0 \%)^{20,23}$. Esses dados indicam a necessidade de programas de intervenção precoce voltados aos maus hábitos de estilo de vida dos estudantes de medicina, como má higiene do sono e uso de bebidas estimulantes durante o dia.

\section{CONCLUSÃO}

Por meio da presente pesquisa, observou-se que a qualidade de sono dos estudantes de Medicina é inferior à da população geral, estando diretamente relacionada à progressão do curso. Foi concluído que os discentes deste estudo têm, em média, menos horas de sono que o restante dos brasileiros.

Mudanças que podem parecer relativamente menores em comparação com a pandemia global têm o potencial para causar drásticas transformações na progressão da carreira de futuros médicos. Como não se sabe muito sobre o impacto, em longo prazo, da Covid-19 na educação ${ }^{31}$ médica, é necessário registrar e estudar o impacto total das mudanças que estão sendo realizadas na metodologia de ensino médico.

Dessa forma, acompanhamento psicológico (por meio de atendimentos remotos, o que facilitaria o acesso aos núcleos de apoio psicológico), ações de promoção de saúde e higiene do sono, estratégias de suporte e debates sobre saúde mental no contexto da Covid-19 devem ser disponibilizados pela instituição aos graduandos em Medicina, visando auxiliá-los em suas necessidades durante o período de graduação e assim aprimorar sua formação profissional e pessoal.

\section{CONTRIBUIÇÃO DOS AUTORES}

Glenia Junqueira Machado Medeiros participou da conceituação do estudo, da curadoria de dados, da análise formal, da metodologia e da administração, supervisão e visualização do projeto. Pedro Fernandes Roma participou da conceituação do estudo, da curadoria de dados, da análise formal, da investigação, da metodologia, da seleção de software, da supervisão, validação e visualização do projeto, e da escrita, revisão e edição do manuscrito. Pedro Henrique Meirelles Ferreira Pinheiro de Matos participou da conceituação do estudo, da curadoria de dados, da análise formal, da metodologia, da visualização do projeto, e da escrita, revisão e edição do manuscrito.

\section{CONFLITO DE INTERESSES}

Declaramos não haver conflito de interesses.

\section{FINANCIAMENTO}

Declaramos não haver financiamento.

\section{REFERÊNCIAS}

1. Niedermeyer E. Historical aspects. In: Niedermeyer E, Silva Fl, editors. Electroencephalography, basic principles, clinical applications and related fields. 5th ed. Baltimore: Lippincot Williams \& Wilkins; 2005. p. 1-15.

2. Reilly T, Atkinson G, Waterhouse JM. Exercise and sport science. Philadelphia: Lippincott Williams \& Wilkins; 2000. p. 351-72.

3. Schmidt RF. Neurofisiologia: Vigília, sono e sonhos. São Paulo; Editora EPU, 1979. p. 316-326.

4. Spiegel K, Leproult R, Cauter E. Impact of sleep debt on physiological rhythms. Rev Neurol (Paris). 2003;159(11):511-20.

5. Kryger M, Roth T, Dement W. History of sleep physiology and medicine: principles and practice of sleep medicine. 4th ed. Philadelphia: Elsevier Saunders; 2005.

6. Morch FL, Toni PM. A influência da privação do sono na qualidade de vida. IX Seminário de Pesquisa, Iniciação Científica; Universidade Tuiuti do Paraná; Curitiba; 2005.

7. Bianchin M, Kapczinski F, Quevedo J, Izquierdo I. Estudo do sono e de seus distúrbios: Bases biológicas dos transtornos psiquiátricos. Porto Alegre: Artmed; 2004.

8. Martinez D. Prática da medicina do sono. São Paulo; Editora BYK; 1999.

9. Roth T, Zammit G, Kushida C, Doghramji K, Mathias S, Wong J. A new questionnaire to detect sleep disorders. Sleep Med. 2002;3(2):99-108.

10. Rodrigues DG, Pelisoli C. Ansiedade em vestibulandos: um estudo exploratório. Rev Psiq Clín. 2008;35(5):171-7.

11. Cerqueira A, Lima M. A formação da identidade do médico: implicações para o ensino de graduação em medicina. Interface Comun Saúde Educ. 2002;6(11):107-16.

12. Raj S, Simpson C, Hopman W, Singer M. Health-related quality of life among final-year medical students. Can Med Assoc J. 2000;162(4):509-10.

13. Alves J, Tenório $M$, Anjos A, Figueroa J. Qualidade de vida em estudantes de medicina no início e final do curso: avaliação pelo Whoqol-Bref. Rev Bras Educ Med. 2010;34(1)91-96.

14. Medeiros A, Lima P, Almondes K, Dias S, Rolim S, Araújo J. Hábitos de sono e desempenho em estudantes de medicina. R. Saúde. 2003;16:49-54.

15. Ahmed MZ, Ahmed O, Aibao Z, Hanbin S, Siyu L, Ahmad A. Epidemic of Covid-19 in China and Associated Psychological Problems. Asian J Psych. 2020;51:e102092. 
16. Wilder-Smith A, Freedman DO. Isolation, quarantine, social distancing and community containment: pivotal role for old-style public health measures in the novel coronavirus (2019-nCoV) outbreak. J Travel Med. 2020;27(2):3-13.

17. Hossain MM, Sultana A, Purohit N. Mental health outcomes of quarantine and isolation for infection prevention: a systematic umbrella review of the global evidence. Epidemiol Health. 2020;42:1-11.

18. Organização Mundial da Saúde. Neurociência do consumo e dependência de substâncias psicoativas. OMS; 2004.

19. Araújo M, Lima A, Alencar A, Araújo T, Fragoso L, Damasceno M. Avaliação da qualidade do sono de estudantes universitários de Fortaleza-CE. Texto contexto - enferm. 2013;22(2):352-60.

20. Cardoso H, Buenoi F, Mata J, Alves A, Jochims I, Vaz I, et al. avaliação da qualidade do sono em estudantes de Medicina. Rev Bras Educ Med. 2009;33(3):349-55.

21. Bertolazi A, Fagondes S, Hoof L, Dartora E, Miozzo I, Barba M, et al. Validation of the Brazilian Portuguese version of the Pittsburgh Sleep Quality Index. Sleep Med. 2011;12(1):70-5.

22. Moraes CAT, Edelmuth DGL, Novo NF, Hübner CK. Qualidade de sono em estudantes de Medicina do método de aprendizado baseado em problemas. Medicina (Ribeirão Preto). 2013;46(4):389-97.

23. Ribeiro CRF, Silva YMGP, Oliveira SMC. O impacto da qualidade do sono na formação médica. Rev Soc Bras Clín Méd. 2014;12(1):8-14.

24. Corrêa CC, Oliveira FK, Pizzamiglio DS, Ortolan EVP, Weber SAT. Sleep quality in medical students: a comparison across the various phases of the medical course. J Bras Pneumol. 2017;43(4):285-9.

25. Poyares D, Tufik S. I Consenso Brasileiro de Insônia. São Paulo: Sociedade Brasileira de Sono; 2003.

26. Valle L, Eduardo L, Valle R, Reimão R. Sono e aprendizagem. Rev. Psicopedagogia. 2009; 26(80):286-90.

27. Segundo L, Cavalcanti Neto B, Paz D, Holanda M. Aspectos relacionados à qualidade do sono em estudantes de medicina. Rev Bras Neurol Psiq. 2017;21(3):213-23.

28. Kim HJ, Kim JH, Park K-D, Choi K-G, Lee HW. A survey of sleep deprivation patterns and their effects on cognitive functions of residents and interns in Korea. Sleep Med. 2011;12(4):390-6.
29. Curcio G, Ferrara M, Gennaro L. Sleep loss, learning capacity and academic performance. Sleep Med Rev. 2006;10(5):323-37.

30. Thomas ML, Sing HC, Belenky G, Holcomb HH, Mayberg HS, Dannals RF, et al. Neural basis of alertness and cognitive performance impairments during sleepiness. I. Effects of $24 \mathrm{~h}$ of sleep deprivation on waking human regional brain activity. Sleep Res. 2000;9(3):335-52.

31. Chang J, Yuan Y, Wang D. Mental health status and its influencing factors among college students during the epidemic of Covid-19. Journal of Southern Medical University. 2020;40(2):171-6.

32. Gulano MR, Moro G, Voglino G, Bert F, Siliquini R. Effects of Covid-19 lockdown on mental health and sleep disturbances in Italy. Int J Environ Res Public Health. 2020 July 2;17(13):47-79.

33. Huang $Y$, Zhao N. Generalized anxiety disorder, depressive symptoms and sleep quality during Covid-19 outbreak in China: a web-based crosssectional survey. Psychiatry Res. 2020;288(11):29-54.

34. Cellini N, Canale N, Mioni G, Costa S. Changes in sleep pattern, sense of time and digital media use during Covid-19 lockdown in Italy. J Sleep Res. 2020;29(4):e13074. doi: 10.1111/jsr.13074.

35. Yuan $S$, Liao $Z$, Huang $H$, Jiang $B$, Zhang $X$, Wang $Y$, et al. Comparison of the indicators of psychological stress in the population of Hubei province and non-endemic provinces in China during two weeks during the coronavirus disease 2019 (Covid-19) outbreak in February 2020. Med Sci Monit. 2020;26:e923767.

36. Wang C, Pan R, Wan X, Tan Y, Xu L, Ho CS, et al. Immediate pschological responses and associated factors during the initial stage of the 2019 coronavirus disease (Covid-19) epidemic among the general population in China. Int J Environ Res Public Health. 2020;17(5):17-29.

37. Etxebarria N, Santamaria M, Gorrochategui M. Stress, anxiety, depression levels in the initial stage of the Covid-19 outbreak in a population sample in the northern Spain. Cad Saude Publica. 2020;36(4):e00054020.

38. Cao W, Fang Z, Hou G, Han M, Xu X, Dong J, et al. The psychlogical impact of the Covid-19 epidemic on college students in China. Pschiatry Res. 2020;287(11):29-34. 